\title{
A saúde vista com outros olhos: Iluminação Hospitalar
}

\author{
Gúliti Ricardo Fagundes Nascimento \\ Arquiteto e Urbanista pela Universidade do Estado de Mato Grosso \\ Pós-graduado em Gerenciamento de Obras pelo Instituto Cuiabano de Educação - ICE \\ Mestrando em Arquitetura, Tecnologia e Cidade - UNICAMP \\ 凶 guliti_ricardo@hotmail.com
}

Recebido em 5 de maio de 2019

Aceito em 5 de dezembro de 2019

\begin{abstract}
Resumo:
A iluminação no ambiente físico hospitalar tem como finalidade contribuir para ambientes mais humanizados, auxiliando no tratamento de pacientes e acelerando sua recuperação, além da qualidade das atividades realizadas pelos profissionais que trabalham e prestam serviços em hospitais. O papel da iluminação na qualificação dos espaços hospitalares e a melhora do estado fisiológico e psicológico dos indivíduos são geralmente ignorados. Sua influência positiva é especialmente importante para os pacientes que se encontram confinados nos quartos de internação. $O$ objetivo deste trabalho é analisar a importância da iluminação natural e artificial para o bem-estar dos usuários em espaços hospitalares, não apenas pacientes como também funcionários e visitantes. Os resultados desde estudo contribuirão no tratamento terapêutico do paciente hospitalizado com ênfase na qualidade e segurança do serviço realizado pelos profissionais da área da saúde de forma mais humanizada. A iluminação tem papel importante no ambiente físico hospitalar, capaz de influenciar no comportamento humano.
\end{abstract}

Palavras-chave: Ambiente físico hospitalar, usuários, iluminação, ambiente humanizado.

\section{Health seen with other eyes: Hospital illumination}

\begin{abstract}
:
The illumination in the hospital's physical environment aims to contribute to more humanized environments, assisting in the treatment of patients and accelerating their recovery, as well as the quality of the activities performed by professionals who work and provide services in hospitals. The role of lighting in the qualification of hospital spaces and the improvement of the physiological and psychological state of individuals are generally ignored. Their positive influence is especially important for patients who are confined in the inpatient rooms. The objective of this work is to analyze the importance of natural and artificial lighting for the well-being of users in hospital spaces, not only patients but also employees and visitors. The results from the study will contribute to the therapeutic treatment of hospitalized patients with an emphasis on the quality and safety of the service performed by health professionals in a more humanized way. Lighting has an important role in the physical hospital environment, capable of influencing human behavior.
\end{abstract}

Keywords: Physical hospital environment, users, lighting, humanized environment. 


\section{La salud vista con otros ojos: Iluminación Hospitalaria}

\section{Resumen:}

La iluminación en el ambiente físico hospitalario tiene como finalidad contribuir a ambientes más humanizados, auxiliando en el tratamiento de pacientes y acelerando su recuperación, además de la calidad de las actividades realizadas por los profesionales que trabajan y prestan servicios en hospitales. El papel de la iluminación en la calificación de los espacios hospitalarios y la mejora del estado fisiológico y psicológico de los individuos son generalmente ignorados. Su influencia positiva es especialmente importante para los pacientes que se encuentran confinados en las habitaciones de internación. El objetivo de este trabajo es analizar la importancia de la iluminación natural y artificial para el bienestar de los usuarios en espacios hospitalarios, no sólo pacientes como también empleados y visitantes. Los resultados desde estudio contribuirán en el tratamiento terapéutico del paciente hospitalizado con énfasis en la calidad y seguridad del servicio realizado por los profesionales del área de la salud de forma más humanizada. La iluminación tiene un papel importante en el ambiente físico hospitalario, capaz de influir en el comportamiento humano.

Palabras-clave: Ambiente físico hospitalario, usuarios, iluminación, ambiente humanizado.

\section{INTRODUÇÃo}

O termo ambiente humanizado nos estabelecimentos de saúde está relacionado à estrutura física espacial do edifício, gerada a partir de projetos de arquitetura, pois esta busca padrões de projeto que proporcionem a satisfação e o bem-estar dos seus usuários, utilizandose de artifícios e de organização em sua ambientação (TOLEDO, 2006). Para Fontes (2004), ao projeto arquitetônico cabe a tarefa de harmonizar e tentar atender em um mesmo espaço físico as distintas realidades postas ao sistema hospitalar, visto que o ambiente tem fundamental importância na qualidade de atendimento ao paciente. A arquitetura hospitalar pode e deve contribuir para o processo de melhoria do usuário, constituindo-se em um instrumento terapêutico.

No Brasil, muito se fala, mas pouco se tem certeza a respeito de humanização hospitalar, pois o emprego deste termo pode muitas vezes ser utilizado para caracterizar a estrutura física de um estabelecimento ou do serviço sobre ele prestado à população (MEDEIROS, 2004).

No tocante, há quem diga que no ramo da assistência médico-hospitalar, o ambiente humanizado é uma prática constante, principalmente porque existe uma ideia de que para atrair pacientes, essa é uma marca garantida com um padrão superior de 
qualidade, já para outros, ambiente humanizado é aquele no qual existem pessoas que prestam trabalhos voluntários (MEDEIROS, 2004).

Os conhecimentos científicos voltados para arquitetura hospitalar proporcionaram um entendimento maior sobre a interferência do meio ambiente na saúde das pessoas, podendo ajudar no tratamento de doenças, sendo importante considerar que a natureza do trabalho da assistência médico-hospitalar vem se transformando ao longo dos anos, agregando-se novos valores em busca de uma melhor qualidade de vida dos pacientes, por meio de mudanças nos aspectos ambientais dos estabelecimentos de saúde, passando a fazer parte da garantia e do cuidado com o paciente no resultado de seu tratamento (ANVISA, 2014).

Para Fontes e Santos et all (2004), a humanização em ambientes de saúde tem sido levada em consideração ao se projetar. Os autores argumentam que:

O conceito de humanização do atendimento tem sido aplicado nos mais recentes projetos em arquitetura da saúde, representando o desdobramento de um novo enfoque, centrado no usuário, que passa a ser entendido de forma holística, como parte de um contexto, e não mais como o conjunto de sintomas e patologias a serem estudadas pelas especialidades médicas. (FONTES E SANTOS et al, 2004, pag.59)

A iluminação é fator imprescindível a ser considerado no momento da concepção do projeto, visto que contribui para determinar a qualidade e percepção do ambiente. À luz devese grande parte da responsabilidade na melhor interação dos usuários com o espaço. Através deste elemento o profissional poderá, por exemplo, ampliar visualmente os ambientes, integrá-lo à natureza e torná-los os mais adequados e confortáveis possíveis (BITENCOURT, 2013).

De acordo com Kasper, Bittencourt e Grau (2009, p. 92).

No Brasil, a maioria dos estabelecimentos de saúde é desprovida de qualquer diferença no tipo de iluminação, que em grande parte a iluminação não segue o que é prescrito pelas normas. Todavia esta não é a maneira adequada, pois cada espaço tem sua necessidade e o seu desempenho. Neste contexto, devem-se verificar os benefícios que a luz traz à saúde, não devendo ser somente tratada como um recurso puramente visual para a execução das atividades nas unidades que compõem o complexo hospitalar (como os procedimentos diversos, os exames, o repouso e a vigília) é importante prever sistemas de iluminação que favoreçam a sua realização com qualidade e segurança. 
A iluminação natural deve ser inserida no edifício com a intenção de melhorar a qualidade física do espaço, pois além de contribuir na recuperação dos pacientes, esta também pode ser usada na redução do consumo energético, diminuindo a necessidade do uso da iluminação artificial. Por fim, a iluminação quando em harmonia pode ajudar na recuperação dos pacientes, trazendo significativos avanços no seu tratamento, bem como para a humanização desses espaços, tornando-os agradáveis e confortáveis (HOREVICZ, 2007).

O papel da iluminação natural e artificial na qualidade dos espaços hospitalares é especialmente importante tendo em vista o estado fragilizado dos pacientes e a permanência na instituição por períodos prolongados, bem como alterações de humor e estado de alerta nos pacientes como ocorre nas áreas de internação. A despeito disso, no país, a concepção dos projetos de iluminação de hospitais se limita à satisfação das iluminâncias mínimas estabelecidas pelas normas, em que a influência positiva da iluminação como a melhoria do estado psicológico e fisiológico dos indivíduos, é geralmente ignorada (VASCONCELOS, 2004, p. 49).

Diante disto, esta pesquisa se propõe a investigar a interferência positiva e negativa da luz no ambiente hospitalar e como esta afeta o sistema visual dos pacientes uma vez que pode ocasionar fadiga, estresse e desconforto visual comprometendo a saúde e o bem-estar do usuário.

\section{DESENVOLVIMENTO}

\section{Iluminação Hospitalar}

A elaboração de um projeto de iluminação para ambientes hospitalares é um processo complexo que deve buscar, invariavelmente, satisfazer à diversidade de critérios técnicos e às compatibilidades físico-funcionais. A solução projetual deve atender prioritariamente às demandas da atividade ali desempenhada, compatibilizando a possibilidade de realização da função assistencial com outros requisitos pertinentes à arquitetura e ao conforto humano (BITENCOURT, 2007, p. 46).

Para se estabelecer a qualidade do ambiente construído para assistência à saúde, há de se considerar os aspectos de conforto visual proporcionado pelos componentes da iluminação com o objetivo de facilitar o desempenho das atividades a serem desenvolvidas. 
Para a Associação Brasileira de Normas Técnicas (ABNT), em sua NBR ISO/CIE 8.995 - 1 Iluminação de ambientes de trabalho (2013, p. 1).:

Uma boa iluminação propicia a visualização do ambiente, permitindo que as pessoas vejam, se movam com segurança e desempenhem tarefas visuais de maneira eficiente, precisa e segura, sem causar fadiga visual e desconforto. A iluminação pode ser natural, artificial ou uma combinação de ambas.

Portanto, o controle do uso da luz e de sua intensidade deve constituir uma preocupação na hora de projetar ambientes hospitalares, o conforto visual do ambiente pode encorajar a ativa consciência na participação da ação terapêutica. $O$ desenho do ambiente deve, portanto, levar em conta as demandas lumínicas do usuário, além da essencialidade das condições naturais do ambiente (ANVISA, 2014).

Em locais destinados à internação, onde o usuário pode permanecer por muitas horas ou dias, as condições de iluminação artificial e a visualização do ambiente externo podem trazer conforto, além da importante percepção ou orientação do tempo em face do ciclo circadiano. Para os ambientes de emergência, salas cirúrgicas, unidades de terapia intensiva e demais áreas críticas, os efeitos do impacto visual provocado pela iluminação excessiva podem resultar em desconfortos emocionais relevantes, produzindo irritação e estresse, emoções que reduzem a qualidade da assistência (ANVISA, 2014).

Para Bitencourt (2007, p. 47) o desenho do espaço, os elementos funcionais e estéticos, a utilização adequada da iluminação natural e artificial, o uso das cores e, naturalmente, os aspectos vinculados ao conforto ambiental, assumem um papel fundamental na aproximação entre a atividade realizada no ambiente e o resultado desta. Esta abordagem ganha relevância quando se observa a sua importância no acolhimento proposto pelos programas de humanização dos ambientes de saúde, seja esse serviço de caráter público ou privado.

\section{A Importância da Iluminação no Ambiente Hospitalar}

Segundo Horevics (2007), a iluminação é um componente essencial na elaboração de projetos arquitetônicos, levando em consideração a iluminação natural e artificial, assim como o uso do ambiente e a atividade exercida naquele determinado espaço físico. Além disso, a iluminação deve atender às demandas de acordo com as diferentes percepções de um 
determinado grupo de usuários (pacientes e profissionais) para que os projetos atendam de uma forma geral as necessidades físicas, psicológicas e funcionais (BINS ELY, 2006).

De acordo com Corbella (2003), a iluminação não deve ser tratada somente como um recurso visual, mas também terapêutico, capaz de trazer benefícios para a saúde do paciente ao proporcionar sensação psicológica do tempo, tanto do ponto de vista cronológico (ciclo circadiano) quanto climático, em que demonstra a redução no tempo de internação quando o paciente tem noção de temporalidade, percepção da variação da luz durante o dia e visão para o exterior (PECCIN, 2002).

Para Mascarello (2005), o uso adequado da iluminação natural também traz vantagens econômicas para o edifício através de uma boa eficiência energética, pois além de permitir o contato visual com o exterior e utilizar adequadamente a luz natural, são fatores fundamentais, tanto para manutenção dos níveis de conforto visual e psicológico do usuário como para aumento da eficiência energética da edificação.

Para Zumtobel (2008), a luz natural é fator dominante durante o dia nestes ambientes, onde se deve prever a demarcação das funções principais com a iluminação artificial adequada para a utilização durante o período noturno. Em locais com pouca iluminação natural, há a possibilidade de se criar, com a iluminação artificial, os efeitos da luz natural diurna com variações nos diversos horários do dia, visto que, tais efeitos podem reduzir a tensão da espera gerando bem-estar, e auxiliando no relaxamento dos acompanhantes. Desse modo, o projeto de iluminação em ambientes hospitalares constitui-se em uma importante etapa do planejamento do edifício como um todo, principalmente se pensado como fator principal para promover a humanização do espaço físico (ZUMTOBEL, 2008).

\section{Impacto da Luz nos Seres Humanos}

Para uma pessoa doente, a luz é como remédio, pois a iluminação dinâmica, projetada para simular as mudanças na luz do dia, apoia os ritmos circadianos dos pacientes, eleva o ânimo e, assim, promove a sua recuperação (ABRAHÃo, 2009).

A luz desempenha um papel fundamental, além de ter um efeito estético é um relogio biológico importante, uma sugestão ou sincronizador para o nosso relógio interno. Numerosos estudos mostram a conexão entre boa iluminação, saúde e bem-estar, e um crescente número de hospitais usa iluminação que não é apenas ergonômico, mas também provoca uma resposta emocional e promove a saúde como forma de tratamento mais eficiente (ABRAHÃO, 2009). 
A retina do olho humano não é composta apenas de bastonetes e cones que predominantemente servem à percepção visual, mas também células ganglionares da retina fotossensíveis intrínsecas (ipRGC). Em 2007, descobriu-se que essas células contêm melanopsina e, portanto, são sensíveis à luz. A sensibilidade máxima para o efeito melanópico da luz a um comprimento de onda de $490 \mathrm{~nm}$ (azul). Isso significa que a melanopsina é especialmente bem estimulada neste comprimento de onda - figura 1 (DIAL, 2016).

Figura 1 - Espectro efetivo para o efeito melanópico da luz Smel $(\lambda)$.

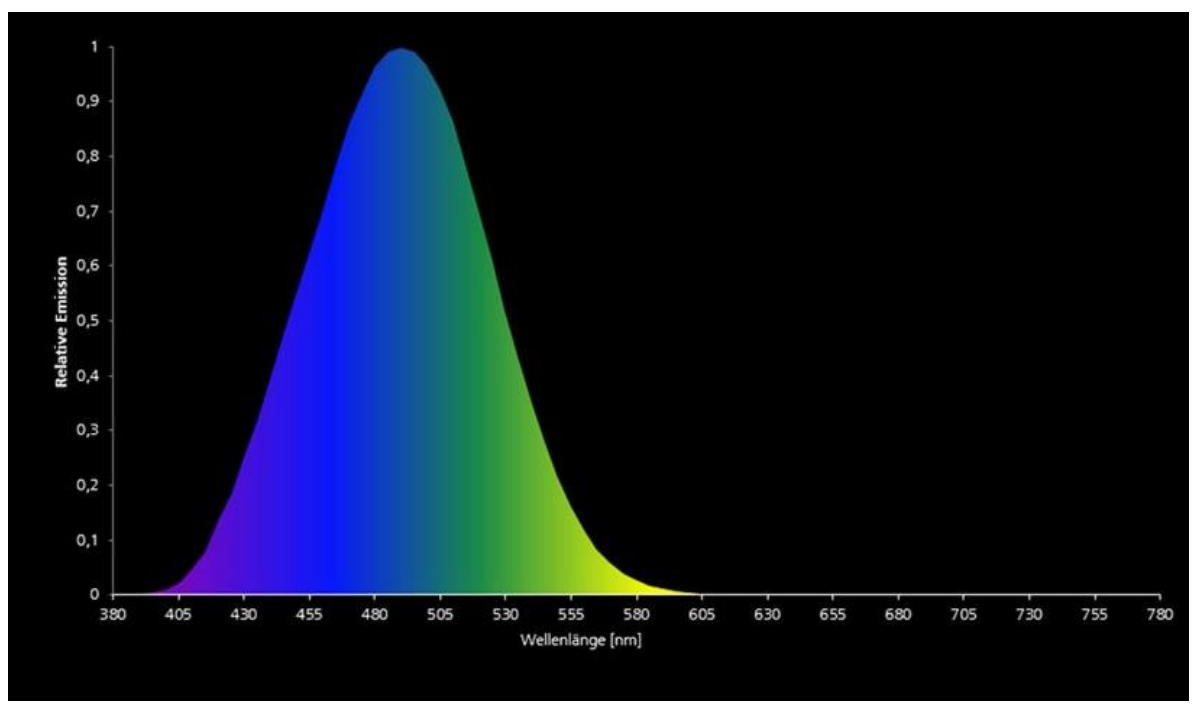

Fonte: DIAL, 2016.

O efeito dessa estimulação é que a supressão da produção do hormônio melatonina ocorre na glândula pineal. O hormônio melatonina desempenha um papel central no ritmo circadiano do homem. Especialmente à noite, quando estamos cansados, nosso sangue tem uma alta concentração de melatonina que cai enquanto dormimos, de modo que o nível de melatonina é consideravelmente menor pela manhã. A luz do dia, com sua alta proporção de luz azul (especialmente pela manhã), também contribui para a supressão da melatonina (DIAL, 2016).

Pesquisas "centradas no humano" realizadas na Alemanha, em que o homem é o foco principal do projeto de iluminação, pretendem imitar uma espécie de progressão da luz do dia com diferentes temperaturas de cor e, por vezes, diferente iluminâncias, a fim de proporcionar uma forma de compensação para os seres humanos que sofrem principalmente de uma falta crônica de luz do dia, tendo como objetivo principal ativar no ser humano a melatonina com cores de luz branca neutra ou fria. Isto pode ser feito com luminárias 
eficientes contendo diferentes temperaturas de cor que podem ser controladas separadamente e são frequentemente chamadas de "branco ajustável" (DIAL, 2016).

\section{METODOLOGIA}

Trata-se de um estudo com abordagem qualitativa de revisão bibliográfica, que visa discutir soluções de conforto ambiental por meio da iluminação hospitalar, como auxílio na melhoria da qualidade física do espaço, e no processo de humanização das unidades hospitalares em geral.

Como a iluminação pode facilitar ou restringir as ações humanas no ambiente hospitalar através do sistema visual, a pesquisa analisou os resultados obtidos considerando a integração dos aspectos qualitativos e quantitativos da luz na qualidade de iluminação, de modo $\mathrm{a}$ atender às exigências humanas e funcionais.

A integração entre a iluminação, os aspectos fisiológicos e os aspectos psicológicos nos permitirão traçar um diagnóstico mais amplo da qualidade da iluminação no ambiente hospitalar.

Nesse sentido pacientes e funcionários se configuram como beneficiários diretos, uma vez que podem alcançar ambientes mais humanizados, com maior qualidade de iluminação, capaz de gerar bem-estar e conforto visual aos mesmos, bem como o melhor desempenho de suas atividades e, consequentemente, gerar ambientes que atendam à necessidade dos seus usuários.

\section{ANÁLISE DE DADOS}

Os primeiros estudos conhecidos sobre a intensidade da luz e ritmos circadianos concluíram que uma iluminância superior a 1000 lux era necessária para a estimulação circadiana (COLE et al., 1995 e ESPIRITU KRIPKE, ANCOLI-ISRAEL, 1994). Segundo Dumont e Beaulieu (2006) e Tenner (2003), hoje se sabe que a exposição a níveis mais baixos de iluminação, como aqueles encontrados usualmente na iluminação interna (raramente superior a 500 lux), já tem um efeito no relógio biológico, mas não está definido se ele é significativo ou suficiente (MARTAU e SCARAZZATO, 2009).

Para Ruger (2006), a luz mais intensa pode influenciar a psicofisiologia instantaneamente ao induzir o sistema endócrino (supressão da melatonina e elevação dos 
níveis de cortisol), a provocar outras mudanças fisiológicas (como a elevação da temperatura corporal, por exemplo) e modificar variáveis psicológicas (redução da sonolência, aumento da atenção).

\begin{abstract}
Essa abrangência de influências da luz faz com que ela se reflita em muitos campos de aplicação, desde a otimização do ambiente de trabalho até o tratamento de pacientes deprimidos. A distribuição espacial da iluminação também é importante do ponto de vista da saúde, porque a incidência da luz sobre a parte superior ou inferior da retina tem importância diferente no efeito biológico medido (MARTAU e SCARAZZATO, 2009).
\end{abstract}

A variabilidade da intensidade da iluminação também é importante, pois isso ocorre na condição natural e ideal constantemente ao longo do dia. O sistema circadiano é sensível às mudanças na luminância do ambiente o que significa que um dado sinal é interpretado no contexto de outras exposições ao claro/escuro dentro das 24 horas do dia, e os seus efeitos no sistema circadiano dependem do resultado da interpretação global (DUMONT e BEAULIEU, 2006).

Contudo, aspectos como o contraste entre as partes mais claras e escuras do dia também parecem ter um impacto significativo na estimulação circadiana. Há evidências de que a sensibilidade à luz do sistema circadiano pode ser regulada pelas iluminâncias as quais os indivíduos estão cronicamente expostos (MARTAU e SCARAZZATO, 2009).

\title{
RESULTADOS
}

Os resultados desde estudo contribuem no tratamento terapêutico do paciente hospitalizado com ênfase na qualidade e segurança do serviço realizado pelos profissionais da área da saúde de forma mais humanizada, e como a iluminação é capaz de influenciar o comportamento físico e psíquico humano, com isso, foi possível correlacionar os aspectos fisiológicos com a iluminação, a partir da análise da quantidade de luz que atinge o campo visual dos pacientes, assim como as possíveis condições de ofuscamento, e ainda, analisar a relação da iluminação com o aspecto psicológico, como estado físico e mental dos pacientes, estresse, ansiedade, e o estado de alerta dos mesmos.

A qualidade da luz recebida é diferente entre a fonte natural e a artificial. Os comprimentos de onda atingem o olho e diferentes profundidades na pele, onde desencadeiam reações fisiológicas de produção de substâncias que organizam o equilíbrio 
metabólico. Sua temperatura de cor correlata (ou aparência da cor) também afeta a estimulação circadiana e a supressão da melatonina em seres humanos (REA et al., 2006; WUNSCH, 2007 e POHL, 2006). Temperaturas de cor mais baixas são menos supressoras da melatonina que as altas, isto é, têm menores efeitos cronobiológicos. Segundo Rea (2006), o que parece claro no momento é que a temperatura de cor não pode ser considerada uma medida métrica simples para caracterizar a eficiência das diferentes fontes de luz para estimular o sistema circadiano, pois a relação entre a supressão da melatonina e temperatura de cor não é linear - figura 2 (MARTAU e SCARAZZATO, 2009).

Figura 2 - Propriedades das fontes de luz (temperatura de cor correlata e temperatura em Celsius) e a intensidade de seu efeito cronobiológico (MARTAU e SCARAZZATO, 2009).

\begin{tabular}{|c|c|c|c|}
\hline \multicolumn{4}{|c|}{ Propriedades das fontes artificiais de iluminaçāo e seu efeito cronobiológico } \\
\hline Fonte de luz & $\begin{array}{l}\text { Temperatura de } \\
\text { cor em Kelvin }\end{array}$ & $\begin{array}{c}\text { Temperatura verdadeira } \\
\text { em Celsius }\end{array}$ & $\begin{array}{l}\text { Efeito } \\
\text { cronobiológico }\end{array}$ \\
\hline LED vermelho & 1000 & $<100$ & $\cdots$ \\
\hline LED Laranja & 1500 & $<100$ & $\cdots$ \\
\hline LED amarelo & 2000 & $<100$ & - \\
\hline Vela & 1500 & 1230 & - \\
\hline Lâmpada incandescente & $2000-2600$ & $1730-2330$ & 0 \\
\hline Lâmpada halógena & $2600-3300$ & $2330-3030$ & + \\
\hline Lâmpada fluorescente & $2700-4000$ & $<100$ & ++ \\
\hline Lâmpada de full spectrum & $4000-6000$ & $<100$ & +++ \\
\hline LED branco & - & $<100$ & ++++ \\
\hline LED azul & - & $<100$ & ++++ \\
\hline
\end{tabular}

Fonte: adaptado de WUNSCH, 2007.

Outro estudo comprovou que a temperatura de cor mais elevada estimula a atividade mental, assim como o sistema nervoso simpático e parassimpático. A sonolência tende a ser mais observada sob a condição de iluminação a 3000K se comparada a 5000K, e a indústria já busca o desenvolvimento de fontes com alta temperatura de cor, sendo que as fontes altamente estimulantes da atividade mental devem ser recebidas com cuidado, uma vez que também podem ser extremamente supressoras da melatonina - figura 3 (MARTAU e SCARAZZATO, 2009). 
Figura 3 - Média da supressão da melatonina (\%) para diferentes temperaturas de cor (em K).

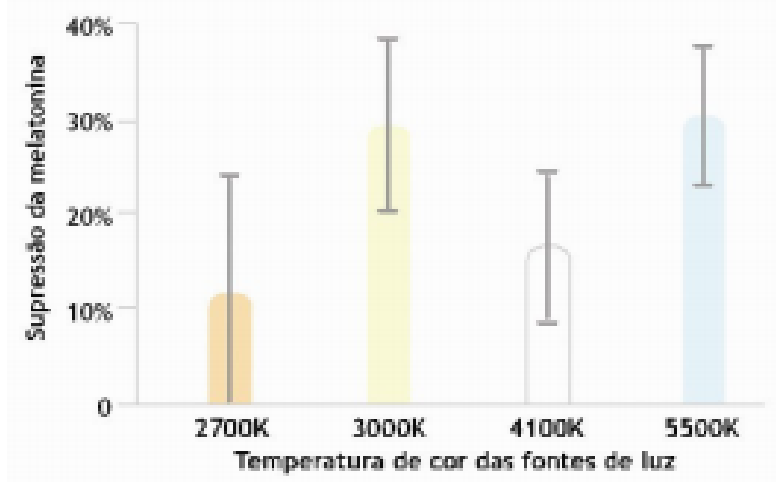

Fonte: adaptado de Figueiro, 2006.

No entanto, a arquitetura hospitalar demanda de profissionais qualificados na área, juntamente com designers de iluminação para uma adequada compreensão dos espaços e de seus usos para que a iluminação possa influenciar beneficamente no tratamento hospitalar e contribuir para a qualidade dos serviços de saúde prestados pelos profissionais (LACERDA, 2016).

\section{CONSIDERAÇÕES FINAIS}

De acordo com as informações apresentadas, pode-se concluir que a arquitetura hospitalar requer profissionais qualificados da área de projetos para uma adequada compreensão dos espaços e de seus usos, para que a iluminação possa influenciar beneficamente no tratamento hospitalar e contribuir na melhora dos pacientes.

A relevância de se considerar a iluminação como fator de humanização possibilita ampliar as chances de atender com qualidade às necessidades da demanda, sendo importante, também, levar em conta as características e condicionantes distinto dos espaços, dos usuários e de suas atividades, extrapolando, se necessário, a mera aplicação dos índices mínimos estabelecidos em normas, na busca por resultados que atendam com maior efetividade às necessidades daqueles que buscam os serviços das unidades hospitalares ou que nela trabalham.

Diante disto, a partir do aspecto da humanização é possível estabelecer parâmetros iniciais para definir projetos adequados em ambientes de saúde, bem como iluminar as 
atividades correlacionadas ao corpo e a mente de maneira que a luz não afete os ritmos metabólicos dos usuários, podendo influenciar a saúde e o bem-estar dos seres humanos.

\section{REFERÊNCIAS}

ABRAHÃO, Júlia. Introdução à ergonomia: da teoria à prática. São Paulo: Blücher, 2009.

ANCOLI-ISRAEL, S.; MARTIN, J. L.; GEHRMAN, P.; SCHOCHAT, T. Effect of light on agitation in institutionalized patients with severe Alzheimer disease. American Journal of Geriatric Pshychiatry, v. 11, n. 2, p. 194-203, 2003.

ASSOCIAÇÃO BRASILEIRA DE NORMAS TÉCNICAS (ABNT). Iluminação de Ambientes de Trabalho. Parte 1: interior. ABNT NBR ISSO/CIE 8.995-1. Rio de Janeiro, abr. 2013, 46 p.

BRASIL. ANVISA. Agência Nacional de Vigilância Sanitária. Conforto Ambiental em Estabelecimentos Assistenciais de Saúde / Tecnologia em Serviços de Saúde. Brasília: $1^{a}$ edição, Agência Nacional de Vigilância Sanitária, 2014.

BRASIL. ANVISA. Agência Nacional de Vigilância Sanitária. Segurança no Ambiente Hospitalar. 2007. Disponível em: < http://www.anvisa.gov.br/>. Acesso em: 03/07/2018.

BINS ELY, V. H. M. Percepção Ambiental e Avaliação Técnico-Funcional em Unidade de Internação Hospitalar. In: XI Encontro Nacional de Tecnologia no Ambiente Construído - A Construção do Futuro. Anais ENTAC. Florianópolis: UFSC, 2006.

BITENCOURT, Fábio. A importância da iluminação e da arquitetura em ambientes hospitalares. São Paulo, Revista Lume, ano IX, n. 59, p. 6-11, dez./2012, jan./2013.

BITENCOURT, Fábio. Iluminação Hospitalar. A luz em ambientes hospitalares como um componente de saúde e conforto humano, revista Lume Arquitetura, edição nº 27, Ago/Set, 2007. Disponível em: http://www.lumearquitetura.com.br/lume/. Acesso em 03/07/2018.

CORBELLA, Oscar; YANNAS, Simos. Em busca de arquitetura sustentável para os trópicos: conforto ambiental. Rio de Janeiro: Revan, 2003.

DUMONT, M.; BEAULIEU, C. Effects of dim and bright work environment on circadian functions. In: CIE EXPERT SYMPOSIUM ON LIGHTING AND HEALTH, 2. Ottawa, 2006. Viena: Commission Internationale de l'Eclairage, p. 46-49, 2006.

ESPIRITU, R. C.; KRIPKE, D. F.; ANCOLI-ISRAEL, S.; MOWEN, M. A.; MASON, W. J.; FELL, R. L.; KLAUBER, M. R.; KAPLAN, O.J. Low illumination experienced by San Diego adults: association with atypical depressive symptoms. Biological Psychiatry, v. 35, p. 403-407, 1994.

FIGUEIRO, M. G. Lighting for Alzheimer's care. In: SYMPOSIUM ON LIGHTING AND HEALTH, 2. Ottawa, 2006. Vienna: Commission Internationale de l Eclairage, p. 69-72, 2006.

HCL - Human Centric Lighting - DIAL. DE. Maio 2016. Disponível em: < https://www.dial.de/en/home/> Acesso em: 02/07/2018. 
HOREVICZ, E. C. Simão. A Humanização em interiores de ambientes hospitalares. Revista Terra e Cultura, $n^{\circ} 45$, Ago/Dez, 2007.

KASPER. A. A.; BITTENCOURT. C. M.; GRAUO. S. A Influência da Iluminação como Fator de Humanização em Ambientes Hospitalares: $O$ Caso das Salas de Espera e Corredores Hospitalares. Simpósio Brasileiro de Qualidade do Projeto no Ambiente Construído - SBQP, USP, 2009.

LACERDA. H. MARINEZ. A Importância da Iluminação no Ambiente Físico Hospitalar, Revista On-Line IPOG/ Dez. 2016.

LICHT.WISSEN 19 - Impact of Light on Human Beings. Disponível em: <https://www.licht.de/en/>. Acesso em 04/07/2018.

MARTAU. T. BETINA; SCARAZZATO. S. PAULO. Impactos não Visuais da Iluminação, Natal, setembro de 2009.

MASCARELLO, Vera Lúcia Dutra. Princípios bioclimáticos e princípios de arquitetura moderna - evidências no edifício hospitalar. Dissertação (Mestrado em Arquitetura) - Universidade do Rio Grande do Sul, Porto Alegre, 2005.

MEDEIROS, LUCIANA. Humanização Hospitalar, Ambiente Físico e Relações Assistenciais: A Percepção de Arquitetos Especialistas. Universidade Federal do Rio Grande do Norte/ Natal, 2004.

PECCIN, Adriana. Iluminação Hospitalar. Estudo de caso: espaços de internação e recuperação. Dissertação (Mestrado em Arquitetura) - Faculdade de Arquitetura, Universidade Federal do Rio Grande do Sul, Porto Alegre, 2002.

POHL, W. Supressão de melatonina por diferentes fontes de luz. Ottawa, 06 Setembro, 2006. Palestra

REA, M. S.; BULLOGH, J. D.; BIERMAN, A.; FIGUEIRO, M. G. Measuring light as a stimulus for the human circadian system. In: SYMPOSIUM ON LIGHTING AND HEALTH, 2. Ottawa, 2006. Viena: Commission Internationale de l'Eclairage, p. 173-177, 2006.

RUGER, M.; GORDIJN, M. C.; BEERSMA, D. G.; DE VRIES, B.; DAAN, S. Time-of-day-dependent effects of bright light exposure on human psychophysiology: comparision of daytime and nightime exposure. American Journal of Physiology, v. 290, p. R1413-R1420, 2006.

FONTES, M. P. Z. Humanização na Arquitetura da saúde: a contribuição do conforto ambiental dos pátios e jardins em clima quente e úmido. Encontro Nacional de Tecnologia do Meio Ambiente Construído - trabalho completo em CD ROM. Anais ENTAC. São Paulo, 2004.

TENNER, A. D. A healthy future for office lighting? Journal of Lighting \& Visual Environment, Japan, v. 27, n. 3, p. 42-46, 2003.

TOLEDO, L. C. Feitos para Curar: Arquitetura Hospitalar e Processo Projetual no Brasil. Rio de Janeiro: ABDEH, 2006, $127 \mathrm{p}$.

WUNSCH, A. Artificial Light and Health. Professional Lighting Design, v. 53, p. 46-49, jan/feb. 2007.

ZUMTOBEL. Lighting Hand Book. Debindorn/Austria, dez. 2008. Disponível em: <http://www.zumtobel.com/healthcare/>. Acesso em: 03/07/2018.

(cc) $\mathrm{Br}$

Este trabalho está licenciado com uma Licença Creative Commons - Atribuição 4.0 Internacional. 